\title{
POLÍTICA DE GOVERNANÇA E ENTREGA DE VALOR EM SAÚDE
}

\author{
GOVERNANCE AND VALUE DELIVERY IN HEALTHCARE
}

\section{Renato Camargos Couto}

Médico, graduado pela Faculdade de Medicina da UFMG.

Especialista em Clínica Médica pelo Hospital das Clínicas da UFMG.

Especialista em Medicina Intensiva pela AMIB.

Doutor em Ciências da Saúde, Infectologia e Medicina Tropical pela Faculdade de Medicina da UFMG.

Professor da pós-graduação em Metodologia DRG (Grupos de Diagnósticos Relacionados) da Faculdade de Ciências Médicas de Minas Gerais - Feluma.

Ex-Professor Associado Departamento de Clínica Médica da Faculdade de Medicina da UFMG.

Ex-Professor do curso de pós-graduação em Ciências da Saúde, Infectologia e Medicina Tropical da Faculdade de Medicina da UFMG.

Coordenador Técnico do curso de Capacitação em Modelo de Remuneração DRG da Faculdade Unimed. Presidente do Grupo IAG Saúde/DRG Brasil®.

\section{Tania Moreira Grillo Pedrosa}

Médica, graduada pela Faculdade de Medicina da UFMG.

Especialista em Clínica Médica pelo Hospital das Clínicas da UFMG.

Especialista em Saúde Ocupacional pela Faculdade de Medicina da UFMG.

Doutora em Ciências da Saúde, Infectologia e Medicina Tropical pela Faculdade de Medicina da UFMG.

Professora da pós-graduação da Faculdade de Ciências Médicas de Minas Gerais - Feluma.

Coordenadora do curso de pós-graduação em Metodologia DRG (Grupos de Diagnósticos Relacionados) da Faculdade de Ciências Médicas de Minas Gerais - Feluma.

Coordenadora Técnica do curso de Capacitação em Modelo de Remuneração DRG da Faculdade Unimed. Presidente do Grupo IAG Saúde/DRG Brasil®. 
A entrega de valor deve ser o propósito dos sistemas de saúde. Essa é a receita para evitar desperdícios e falhas, que prejudicam a assistência ao paciente e os resultados alcançados pelas instituições do setor. Valor em saúde é determinado pela qualidade assistencial dividida pelo custo, alinhado a uma experiência positiva do paciente em sua trajetória no sistema de saúde (1) (2). O inverso de valor, portanto, é desperdício.

Para chegar a um índice positivo, é preciso focar nos 4 alvos assistenciais para aumentar a qualidade e eliminar o desperdício: uso eficiente do leito hospitalar; aumento da segurança assistencial; redução de internações evitáveis; diminuição de readmissões hospitalares não planejadas (3) (4).

As falhas de entrega de valor consomem 2,5\% do Produto Interno Bruto (PIB) americano, conforme levantamento de Berwick e Hackbarth. Isso significa que a eliminação de 50\% do desperdício do sistema de saúde dos Estados Unidos economizaria 1 trilhão de dólares $(5)$.

Uma assistência adequada à atenção primária e um sistema de emergência resolutivo podem reduzir as chamadas internações por condições sensíveis à atenção primária (ICSAP). Hospitalizações cirúrgicas podem, da mesma forma, ser evitadas. Um serviço ambulatorial resolutivo integrado a uma transição de cuidados para o domicílio e incentivos econômicos para médicos e hospitais têm demonstrado elevada efetividade na ambulatorização de procedimentos cirúrgicos (3) (4).

No mundo, há ineficiência no uso do leito hospitalar (1). O aumento da permanência, além do esperado pela complexidade clínica, é determinado por falhas no complexo processo assistencial hospitalar; pela burocracia fútil nas relações entre hospital e operadora, como a demora para autorizações; por falhas nas relações com a família e o paciente; pela ausência de recursos extra-hospitalares para continuidade de cuidados; e por problemas jurídicos e sociais (3) (4)

Eventos adversos, que se manifestam ou agravam após a alta e a transição inadequada do cuidado hospitalar para o nível ambulatorial, são capazes de determinar reinternações precoces potencialmente preveníveis. Por sua vez, a insegurança assistencial hospitalar determina uma pandemia de mortes, sequelas e aumento de custos. É uma das causas mais frequentes de óbitos nos Estados Unidos (6). No Brasil, em 2017, estima-se que 36.174 falecimentos foram atribuíveis aos eventos adversos graves e consumiram R \$ 10,6 bilhões na saúde suplementar, de acordo com o II Anuário da Segurança Assistencial Hospitalar no Brasil (7). 
Para medir o valor entregue pelos sistemas de saúde suplementar e público brasileiro estudamos o banco de dados secundário de todas as altas hospitalares ocorridas no período entre $1^{\text {o }}$ de agosto de 2018 e 31 de julho de 2019 de 129 operadoras de saúde suplementar e SUS, que cobrem 10,586 milhões de vidas e que utilizam o software DRG Brasil@ para fins gerenciais (4). O estudo avaliou: resolutividade da atenção primária; segurança assistencial hospitalar; segurança na transição de cuidado do hospital para o domicílio; a eficiência do uso do leito hospitalar $\mathrm{O}$ desperdício potencialmente modificável pela entrega de valor foi equivalente a $37,7 \%$ de todas as diárias hospitalares consumidas para tratar os pacientes da população estudada (4).

Uma política efetiva de governança clínica do sistema de saúde brasileiro baseada na entrega de valor para a sociedade e demais partes interessadas é boa para todos. O paciente pode ter seus danos físicos e psicológicos, além de seus custos, reduzidos. A sociedade pode garantir mais acesso ao sistema de saúde e aumentar a competitividade de sua economia. Os financiadores do sistema de saúde podem alcançar o triplo objetivo: melhor atendimento aos indivíduos; melhor saúde das populações; menor custo per capita, com preservação dos recursos preciosos para sua sustentabilidade. E, à medida que as organizações prestadoras de serviço de saúde mudam seu foco de volume para valor, a redução do desperdício cria a oportunidade de compartilhamento de recursos com médicos e rede hospitalar (8).

Um sistema de saúde para entrega de valor deve alinhar seu modelo assistencial e remuneratório à segurança, eficácia, eficiência, centralidade do paciente, garantia de acesso oportuno e equitativo. $\mathrm{O}$ modelo de governança deve ser baseado na ciência e garantir ao paciente a assistência contínua, personalizada, com transparência para escolhas conscientes.

Para construir um sistema de saúde baseado em valor, é necessário o envolvimento de todas as partes interessadas: o Estado, os líderes, as agências reguladoras do governo, as parcerias público-privadas, as organizações de saúde (financiadoras e prestadoras), os pesquisadores, as associações profissionais e de clientes, os educadores, a força de trabalho, os pacientes e as famílias (4). O controle do desperdício pela maior entrega de valor pelo sistema de saúde brasileiro poderia mitigar as limitações de acesso à assistência hospitalar, assim como poderia disponibilizar recursos para outras necessidades do sistema de saúde brasileiro.

Mais segurança para o paciente, maior acesso ao sistema de saúde pela população, entrega de uma assistência de qualidade a um menor custo para organizações. Esse o propósito. 


\section{REFERÊNCIAS}

1. PORTER, M.E. What is value in health care? N Engl J Med, v.363, p.2477-81, 2010.

2. STIEFEL, M.; NOLAN, K. A Guide to Measuring the Triple Aim: Population Health, Experience of Care, and Per Capita Cost. IHI Innovation Series white paper. Cambridge, Massachusetts: Institute for Healthcare Improvement, 2012.

3. COUTO, R.C.; PEDROSA, T.M.G. Diretrizes para um sistema de saúde baseado em valor. [publicação online]. Valor em Saúde Brasil. [S.d.]. Disponível em: https://bit.ly/2TwMjLb. Acesso em: 26 set. 2020.

4. COUTO, R.C.; PEDROSA, T.M.G. Índice de Valor do Sistema de Saúde Brasileiro. Relevância, metodologia e resultados. [publicação online]. Valor em Saúde Brasil. [S.d.]. Disponível em: https://bit.ly/2TwMjLb. Acesso em: 26 set. 2020.

5. BERWICK, D.M.; HACKBARTH AD. Eliminating waste in US health care. Journal of the American Medical Association, v.307, n.14, p.1513-1516, 2012.

6. MAKARY MA, DANIEL M. Medical error - the third leading cause of death in the US. $B M J$, v.353, n.2139, p.1-5, 2016.

7. COUTO RC, PEDROSA TMG, ROBERTO BAD, DAIBERT PB, et al. II Anuário da Segurança Assistencial Hospitalar no Brasil. Propondo as Prioridades Nacionais. Belo Horizonte - 2018. Disponível em: https://bit.ly/3jyQz7r. Acesso em: 20 julho 2020.

8. COUTO RC, PEDROSA TMG. Remuneração baseada em valor. Conceitos e métricas. [publicação online]. Valor em Saúde Brasil. [S.d.]. Disponível em: https://bit.ly/2TwMjLb. Acesso em: 26 set. 2020. 